\title{
Long Life Electrochemical Diodes for Continuous Electrowetting
}

Mehdi Khodayari

Georgia Institute of Technology

Ben Hahne

Nathan B. Crane

Brigham Young University - Provo, nbcrane@byu.edu

Follow this and additional works at: https://scholarsarchive.byu.edu/facpub

Part of the Electro-Mechanical Systems Commons, and the Other Mechanical Engineering Commons

\section{Original Publication Citation}

M. Khodayari, B. Hahne, and N. B. Crane, "Long Life Electrochemical Diodes for Continuous Electrowetting," Journal of The Electrochemical Society, Vol 162 (2014) no 6: E105-E111, DOI: 10.1149/2.008406jes.

\section{BYU ScholarsArchive Citation}

Khodayari, Mehdi; Hahne, Ben; and Crane, Nathan B., "Long Life Electrochemical Diodes for Continuous Electrowetting" (2014). Faculty Publications. 5367.

https://scholarsarchive.byu.edu/facpub/5367

This Peer-Reviewed Article is brought to you for free and open access by BYU ScholarsArchive. It has been accepted for inclusion in Faculty Publications by an authorized administrator of BYU ScholarsArchive. For more information, please contact ellen_amatangelo@byu.edu. 


\title{
Long Life Electrochemical Diodes for Continuous Electrowetting
}

\author{
Mehdi Khodayari ${ }^{1}$, Ben Hahne ${ }^{2}$, Nathan B Crane ${ }^{3}$ \\ ${ }^{1}$ School of Chemistry and Biochemistry, Georgia Institute of Technology, 901 Atlantic \\ Drive, Atlanta, GA 30332, USA \\ ${ }^{2}$ Aspen Technology, 100 Innovative Way, Nashua, NH $03062^{3}$ University of South Florida, \\ 34202 E. Fowler Ave ENB 118, Tampa, FL 33620, ncrane@usf.edu
}

\section{Abstract}

The rate of electrochemical reactions in some systems varies with the polarity of the overpotential on the working electrode, introducing an electrochemical diode-like behavior with enly anat the electrode-/electrolyte interface. However, with repeated bipolar cycling, the electrochemical current damages the electrodes. We have employed sequential (connected in series) electrochemical diodes in series with opposing polarities to reduce the diode current while charging a capacitive circuit. We have previously used this eapacitvecapacitive circuit arrangement to actuate aqueous droplets continuously using the electrowetting (EW) effect. In this study, the performance of electrochemical diodes under repeated voltage cycles is investigated. Aluminum and titanium electrodes in contact with three electrolyte solutionsnamely $\left(0.1 \mathrm{M}\right.$ citric acid, $0.1 \mathrm{M} \mathrm{Na}_{2} \mathrm{SO}_{4}$ and $\left.0.1 \mathrm{M} \mathrm{NaOH}\right)$ are employed. The diode behavior of electrochemical systems with single diodes and diode pairs is compared. A coefficient of continuous EWelectrowetting (CEW) actuation (referred to as actuation coefficient) is introduced. Actuation coefficient varies between zero (no actuation) and one-At one, the highest lateral force is applied on the droplet_equivalent to arounded droplet EW actuatoractuation at the same voltage-Our experimental). Experimental results indicateshow that titanium 
pessessesmaintains higher actuation coefficients (between 0.6 to-_0.8) than aluminum (between zero to-0-0.6). The actuation coefficients of titanium increasesincrease over the first trials and remain stable when the test voltage is swept betweenunder alternating $-50 \mathrm{~V}$ toand $+50 \mathrm{~V}$ inputs for 2000 trials (experiment duration).

\section{Introduction}

In contact with other phases (gas, liquid, solid), liquids take a shape that minimizes the free energy (1-2) balancing gravitational and interfacial energy to determine droplet shapes (3). However, aqueous droplets smaller than $80 \mu \mathrm{l}$ (without surfactants) form a spherical cap as surface energy dominates the gravitational energy (4-5). For a droplet on a solid surface, the droplet shape can be defined by the contact angle of the droplet at the Three Phase Contact Line (TCL), the contact line-where the three phases meet.

Electrowetting $(\mathrm{EW})$ is an electromechanical phenomenaphenomenon in which the wetted area and apparent contact angle (and droplet shape/contact area) can be changed by applying an electric potential difference between the droplet and fsubstrateelectrode interface-(6-7). Typically, the substrate contains an electrode is covered with a dielectric to reduce electrochemical reactions. This arrangement, referred to as Electrowetting on Dielectric (EWOD), is the basis of this study. Below a limiting saturation voltage, the apparent wetting angle is given as (8):

$$
\cos \theta_{1}=\cos \theta_{0}+\varepsilon_{0} \varepsilon_{r} V^{2} / 2 \delta \gamma_{L O}
$$

where $\theta_{0}$ and $\theta_{1}$ are the initial and electrowetting droplet angles, $V$ is the applied voltage, $\gamma_{L O}$ is the surface energy between the droplet and the second phase, $\delta$ is the dielectric thickness, and 
$\varepsilon_{0} \varepsilon_{r}$ the dielectric permittivity. The electrostatic force/length on the droplet close to TCL (6-7) is calculated as follows:

$$
\vec{F}=\varepsilon_{0} \varepsilon_{r} \cdot \overrightarrow{V^{2}} / 2 \delta
$$

The electrostatic force is perpendicular to the droplet surface and is only applied on the droplet surface right above the contact line, which vanishes asymptotically towards TCL (9-10). Therefore, local contact angle remains constant at TCL while the apparent contact angle changes (11-12). EWOD has found multiple applications such as microprocessor cooling (13), micromechanical systems (14), electrowetting lenses (15-17), reflective displays (18-19), energy harvesting (20), and lab on chip diagnostics (21-27).

Typically, electrowetting is performed by placing a droplet on top of a an electrode coated with a hydrophobic substratedielectric. A and applying a potential difference is applied between the droplet and the substrateelectrode. In electrowetting with a continuous substrateuniform electrode voltage, the apparent contact angle variation would be identical all over the perimeter. As a result, upon voltage application, contact angle changes without lateral droplet tateral movements (7). However, if we apply a nonuniform voltage on the droplet, the net force on TCL would be nonzero, causing a planar movement of the droplet. Traditionally this is accomplished using a series of different electrodes. Motion is in the direction of the electrode with the higher voltage magnitude relative to the droplet. There are minimal differences between voltage polaritiesy dependence-(28-29). However, in bidirectional electrowetting the diode-like properties of some electrode/electrolyte systems are used to create an EW response with a-strong voltage polarity dependence (30).

In bidirectional electrowetting, the lateral movement of the droplet occurs on two separate plates-electrodes below the droplet. Continuous electrowetting (CEW) integrates this effect on a 
continuous resister to create a continuous voltage gradient along the substrateelectrode just one electrode pairone long resistive electrode (31). In CEW, the diodelike behavior atthere are localized dielectric holes where the electrodes directly contact the electrolyte to create a diodelike response. This causes an asymmetric potential distribution so that the potential at the reverse biased spot is higher than the forward biased spot. The forward biased diode at the low potential side of the droplet has a relatively small voltage difference relative to the surrounding electrode. However, a diode on the higher voltage location will be reversed biased. Since the droplet is a conducting fluid and the current (limited by the reverse biased diode) is relatively small, the voltage is assumed to be uniform through the droplet. Thus, the droplet potential will be close to the electrode potential at the forward biased diode. Due to the continuous voltage gradient in the high resistivity electrode, there will be a large potential difference between the droplet and the electrode on the high potential side of the droplet. This creates an imbalance in the electrowetting forces around the droplet to actuate the droplet toward the higher potential.

The droplet moves towards the spot with higher potential. The droplet can be moved continuously on the substrate-as shown schematically in Figure 1. This simplifies motion control by eliminating the need for numerous electrodes. This is particularly advantageous for $2 \mathrm{D}$ motion by eliminating the need for many independent electrodes in order to move droplets.

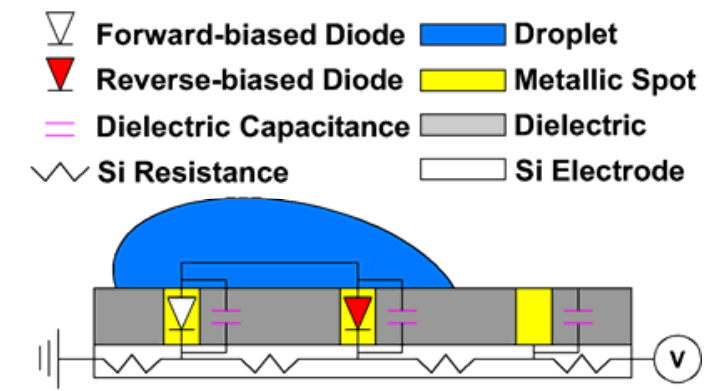

Figure 1. Schematic of continuous electrowetting. 


\subsubsection{Mathematical Model of CEW}

This work will consider the basic case in which the droplet covers two diodes as illustrated in Figure 1. In $\mathrm{CEW}$, to find the net lateral force on the droplet, we integrate the lateral force on the perimeter of the droplet/substrateelectrode interface (Figure 2). The total lateral force can be obtained as follows:

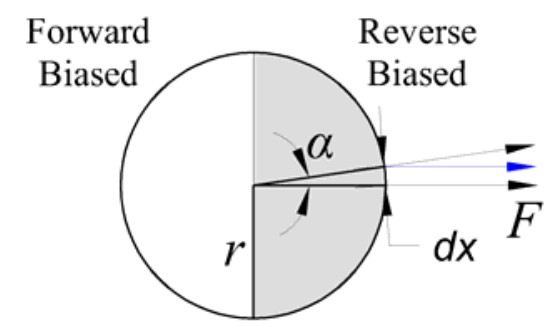

Figure 2. Idealized droplet geometry for estimation of net lateral force in CEW (droplet view from top).

$$
\overrightarrow{d F}_{t, \alpha}=\frac{\varepsilon \cdot\left(V_{\alpha}-V_{d r o p}\right)^{2}}{2 d} \cos \alpha \cdot r \cdot d \alpha
$$

The variables $\alpha$, and $r$ are respectively the angle between two force vectors, and the droplet radius. The variable $V_{\alpha}$ is the substratedroplet to electrode voltage difference on the perimeter of the droplet/substrate interface at the angle $\alpha . V_{d r o p}$ is the droplet voltage, which is a function of the electrical performance of the diodes. The substrateelectrode voltage at the trailing edge of the droplet is taken as zero. $V_{\text {drop }}$ is approximately the turn on voltage of the diodes. Here, $F_{t, \alpha}$ is the force on an arc of the droplet perimeter with the angle of $\alpha$ as shown in Figure 2.

Using a continuous resister, the substrateelectrode voltage changes from zero (at the trailing edge of the droplet) to $V_{\max }$ (at the leading edge of the droplet) linearly by distance. Hence, $V_{\alpha}$ is related to $\alpha$ as follows:

$$
V_{\alpha}=(\cos \alpha+1) \cdot\left(\frac{V_{\max }}{2}\right)
$$

Combining equation 3 and 4, 


$$
\begin{aligned}
& \vec{F}_{t}=\int_{0}^{2 \pi}\left(\frac{\varepsilon \cdot r}{2 d}\left[(\cos \alpha+1) \cdot\left(\frac{V_{\max }}{2}\right)-V_{d r o p}\right]^{2} \cos \alpha\right) \cdot d \alpha \\
& \vec{F}_{t}=\frac{\varepsilon \cdot \pi \cdot r}{4 d} \cdot V_{\max }\left(V_{\max }-2 V_{d r o p}\right)
\end{aligned}
$$

Here, $F_{t}$ is the total lateral force on the droplet. Equation 5 shows how the total lateral force in CEW $\left(F_{t}\right)$ has a linear relationship with $r$ (the droplet radius) and is decreased by the droplet

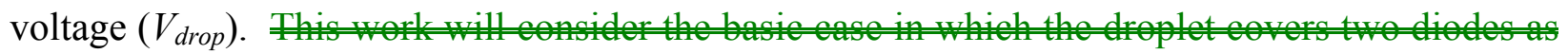
illestated in Figure 1.

The droplet voltage $\left(V_{\text {drop }}\right)$ varies with the metal/electrolyte combinations and the objective of this study is to find metal/electrolyte combinations that produce the highest force that can be maintained over repeated cycling. In the ideal casebest conditions, electrolyte/metal systems act like ideal diodes $(\mathrm{V}>0 \rightarrow \mathrm{R}=$ infinity, $\mathrm{V}<0 \rightarrow \mathrm{R}=0)$. In such a case, $V_{\text {drop }}$ would be close to zero when $V_{\max }$ is positive and $V_{\text {drop }}$ would be close to $V_{\max }$ when $V_{\max }$ is negative, so:

$$
\vec{F}_{t}= \pm \frac{\varepsilon \cdot \pi \cdot r}{4 d} \cdot V_{\max }^{2}
$$

In equation $6, F_{t}$ is either positive or negative. $F_{t}$ is positive when the potential is highest on the right side (Figure 2) and vice versa. Additionally, with a constant droplet/second phase surface tension, lateral electrostatic force is_may reach a maximum atdue to -the electrowetting saturation $v$ oltage, irrespective though this has not been experimentally verified. This peak force would likely be independent of $d$ (the dielectric thickness) and $\varepsilon$ (dielectric constant) (32). The maximum lateral force can be improved by choosing an ambient fluid such as air to increase the droplet/ambient surface energy.

The $V_{d r o p}$ is also affected by the location of the droplet to the nearest diode and the spacing of the diodes. Since this study focuses on electrochemical effects, the diodes are assumed to be ideally situated at the extreme leading and trailing edges of the droplet. A criteriaA criterion that 
relates the diode performance to the efficiency of continuous EW actuation is obtained by dividing equation 5 by equation 6 as follows:

$$
\eta_{\text {actuation }}=\frac{\frac{\varepsilon \cdot \pi \cdot r}{4 d} \cdot V_{\max }\left(V_{\max }-2 V_{d r o p}\right)}{\frac{\varepsilon \cdot \pi \cdot r}{4 d} \cdot V_{\max }^{2}}=1-2 \frac{V_{d r o p}}{V_{\max }}
$$

The $\eta_{\text {actuation }}$ is the coefficient of continuous EW actuation, which varies between zero and one. When $\eta_{\text {actuation }}$ is one, the continuous EW configuration applies the same force as a grounded droplet with $V=V_{\text {max }} . V_{\text {drop }}=V_{\text {left }}$ for one voltage polarity and $V_{\text {drop }}=V_{\text {right }}$ for the other polarity. We experimentally investigate the diode behavior of the metallic spots in different electrode/electrolyte combinations. The diode response characteristics are then used to calculate

$\eta_{\text {actuation }}$ for each combination. The best electrode/electrolyte combinations are recommended for CEW devices.

\section{Experiments}

First, a single diode is characterized using a substrate consisting of $\mathrm{Si} / \mathrm{SiO}_{2} / \mathrm{Metallic}$ Layer with a $50 \mathrm{~nm}$ spin-coated Cytop layer. The thin Cytop layers are permeable so that the electrolyte/electrodes make contact (33). A $5 \mu 1$ droplet is placed on the substrate and a platinum auxiliary electrode placed in the droplet. The potential difference of the substrateelectrode was ramped to $+50 \mathrm{~V}$ down to $-50 \mathrm{~V}$ and back to $0 \mathrm{~V}$ with a frequency of $1 \mathrm{~Hz}$ for the whole cycle while the droplet was grounded. The current is measured concurrently. In this study, all the current and voltage measurements are performed using a Keithley 2612A SourceMeter. Six different electrode/electrolyte combinations are used in this test, namely $\mathrm{Al} / \mathrm{Na}_{2} \mathrm{SO}_{4}, \mathrm{Al} /$ citric acid, $\mathrm{Al} / \mathrm{NaOH}, \mathrm{Ti} / \mathrm{Na}_{2} \mathrm{SO}_{4}, \mathrm{Ti} /$ citric acid, and $\mathrm{Ti} / \mathrm{NaOH}$. 
To characterize diode pairs, the electric potential of the metallic spots was to be measured directly without $\mathrm{IR}$-voltage drop through thea resistive substrateelectrode used in CEW. To do so, pairs of metallic spots are evaluated using a test that simulates two consecutive metallic spots (diodes) in CEW. In this test, metallic electrodes on $\mathrm{SiO}_{2}$ substrates are spin-coated with a $50 \mathrm{~nm}$ Cytop layer. The wafer is divided into two pieces. Two tubes with a diameter of $4.5 \mathrm{~mm}$ are bonded to the substrateswafers and filled with electrolyte solution to create the electrochemical diodes. The two electrolyte-filled tubes are connected with an activated titanium auxiliary electrode as illustrated in Figure 3.

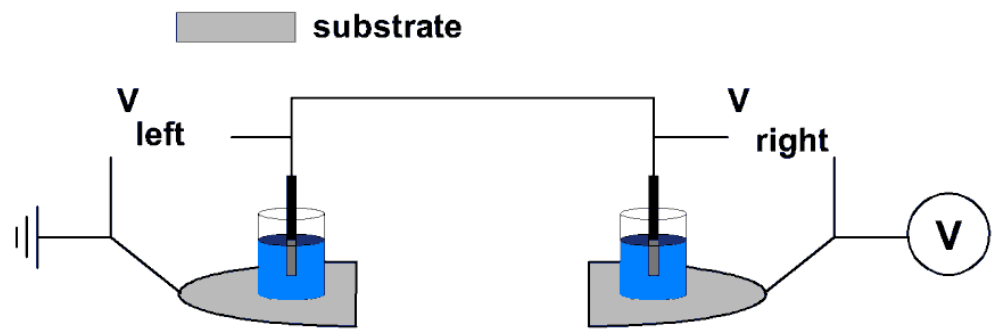

Figure 3. Schematic of experimental setup to measure the metallic spots potential difference in Continuous Electrowetting.

In CEW, the metallic spots are connected via the droplet from top and via the high resistivity silicon from bottom as illustrated in Figure 1. In these tests, the silicon substrateelectrode in CEW is replaced by fixed voltage difference between the two silicon pieces because the substrateselectrodes are only connected through the electrolyte solution in the tubes. In CEW, the other regions have little impact on the electric potential of the stbstrateelectrode because it is covered with a $\mathrm{SiO}_{2}$ dielectric layer which prevents electrochemical reactions $(29,34)$. Therefore, the experiment setup in this study represents CEW with two consecutive metallic spots in contact with a droplet.

A triangular voltage $(1 \mathrm{~Hz})$ is applied between $+50 \mathrm{~V}$ and $-50 \mathrm{~V}$ and the droplet potential $\left(V_{l e f t}\right)$ is measured concurrently. For positive applied voltages, $V_{\text {right }}$ is then calculated as follows: 


$$
\begin{gathered}
V_{\text {left }}=V_{\text {drop }} \\
V_{\text {right }}=V_{\text {applied }}-V_{\text {drop }}
\end{gathered}
$$

where $V_{\text {left }}, V_{\text {applied, }}$, and $V_{\text {right }}$ are respectively the measured voltage (on the left hand of Figure 3 ), applied voltage, and calculated voltage (on the right hand of Figure 3). For negative applied voltges, $V_{\text {left }}$ and $V_{\text {right }}$ are swapped in the equation. Each configuration was tested for at least 500 cycles. The average of trial number 100-102 trial are reported to show the system after the initial break-in period. Additionally, the stability of the response over the entire cycle testing (500 trials with aluminum electrode and 2000 trials with titanium electrode) was evaluated. In all measurements, the test is paused for seven seconds between trials in order to allow any possible trapped charges to relax. The consistency of $\eta_{\text {actuation }}$ in different electrode/electrolyte combinations is compared.

\section{Results and Discussion}
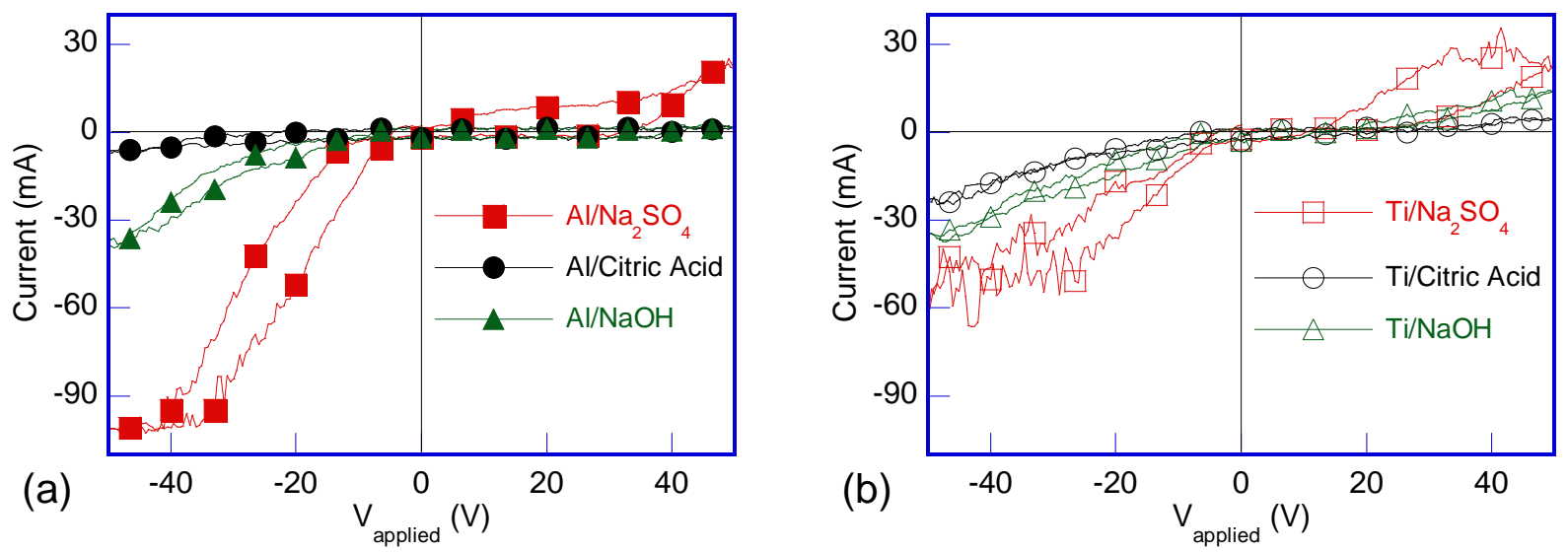

Figure 4. Diode behavior of eight different electrode/electrolyte combinations. In these tests, the droplet is grounded via a platinum auxiliary electrode and the electrode potential is ramped to $+50 \mathrm{~V}$ down to $50 \mathrm{~V}$ and back to $0 \mathrm{~V}$. A $5 \mu \mathrm{l}$ droplet is used in each test. The period of the whole cycle is $1 \mathrm{~s}$.

All electrode/electrolyte combinations behave as diodes to some extent though the aluminum/citric acid exhibits very poor diode behavior as seen in Figure 4. This is seen as higher 
currents for negative applied voltages than for positive voltages in all materials. There is generally some hysteresis in the current/voltage curves that may be indicative of formation of oxides on the electrodes.

The tests with $\mathrm{Na}_{2} \mathrm{SO}_{4}$ have the highest currents with both aluminum and titanium electrodes for forward- and reverse-biased voltages. This could be related to a relatively high charge transfer of $\mathrm{Na}^{+}$cations through the alumina and titania pores, which decreases the potential drop through the oxides and improves the electrochemical charge transfers at the electrodes. With $\mathrm{NaOH}$, the forward current is lower. Here, $\mathrm{Na}^{+}$cations also assistsassist the cathodic charge transfer, but their concentration is half of that with $\mathrm{Na}_{2} \mathrm{SO}_{4}$, which can be the reason of lower forward current. At reverse biased voltages, the tests with $\mathrm{Na}_{2} \mathrm{SO}_{4}$ have the highest currents with both aluminum and titanium electrodes, yet their corresponding I-V curves indicate diode characteristics. One possible reason for the high magnitude of currents with $\mathrm{Na}_{2} \mathrm{SO}_{4}$ can be the porous structure of aluminum oxide and titanium oxide, which paves the way for anions to reach the electrodes surface with subsequent electrochemical reactions. However, in CEW devices, the current is limited by the pairing of two diodes with opposite bias to reduce the current through both the forward and reverse biased sides.

During each diode pair test, $V_{\text {right }}$ and $V_{\text {left }}$ vary in a complementary pattern. In the subsequent graphs (Figure 5), the $V_{\text {left }}$ and $V_{\text {right }}$ are plotted against the applied voltage $\left(V_{\text {app }}\right)$ for an ideal linear diode model $\left(V_{\text {TurnON }}=0\right.$, zero forward-biased resistance, and infinite reversebiased resistance). While an exponential diode better fits the electrochemical diode behavior, the linear model provides a useful reference. 
Electrolytes strongly affect the formation of passivation layers during oxidation. Hence, they change diode behavior of electrodes in our tests. Figure $5 \mathrm{a}$ and $\mathrm{b}$ show $V_{\text {left }}$ and $V_{\text {right }}$ of aluminum electrodes with three different electrolyte solutions, namely $0.1 \mathrm{M}$ citric acid, $0.1 \mathrm{M}$ $\mathrm{N}_{2} \mathrm{SO}_{4}, 0.1 \mathrm{M} \mathrm{NaOH}$. Each fluid shows similar performance. Note the hysteresis between the increasing and decreasing voltage magnitudes. Decreasing magnitudes better approximate the ideal diode.
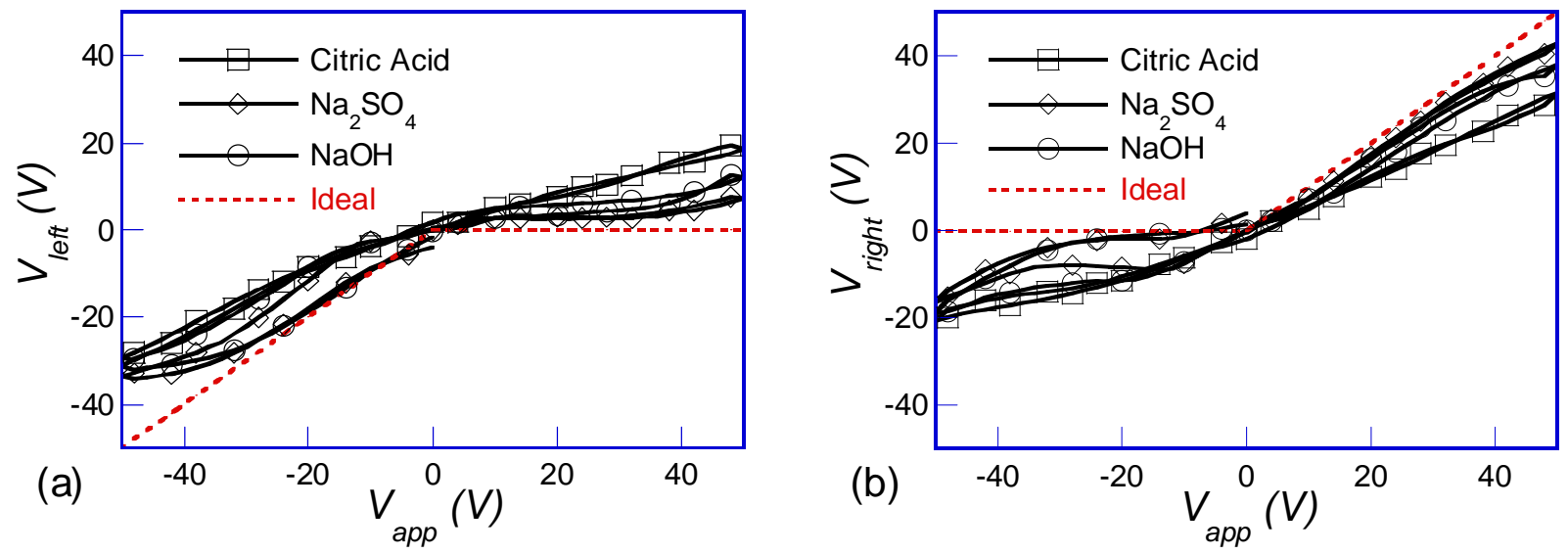

Figure 5. Impact of electrolytes on the (a) $V_{\text {left }}$ and (b) $V_{\text {right }}$ behavior in the electrode pair tests using aluminum electrodes. The $V_{\text {left }}$ and $V_{\text {right }}$ are plotted versus the applied voltage. The dashed (red) lines show the simulated $V_{\text {left }}$ and $V_{\text {right }}$ behavior with the linear ideal. $0.1 \mathrm{M}$ citric acid, $0.1 \mathrm{M} \mathrm{Na}_{2} \mathrm{SO}_{4}, 0.1 \mathrm{M}$ $\mathrm{NaOH}$ are used as electrolyte solutions.

The $V_{\text {left }}$ and $V_{\text {right }}$ curves show some differences. For better comparison among the test results in the electrode pair measurements with aluminum electrode, the $\eta_{\text {actuation }}$ values are plotted versus $V_{a p p}$ as shown in Figure 6Figure 6. Values of $\eta_{\text {actuation }}$ are calculated from the voltages in Figure 5. 


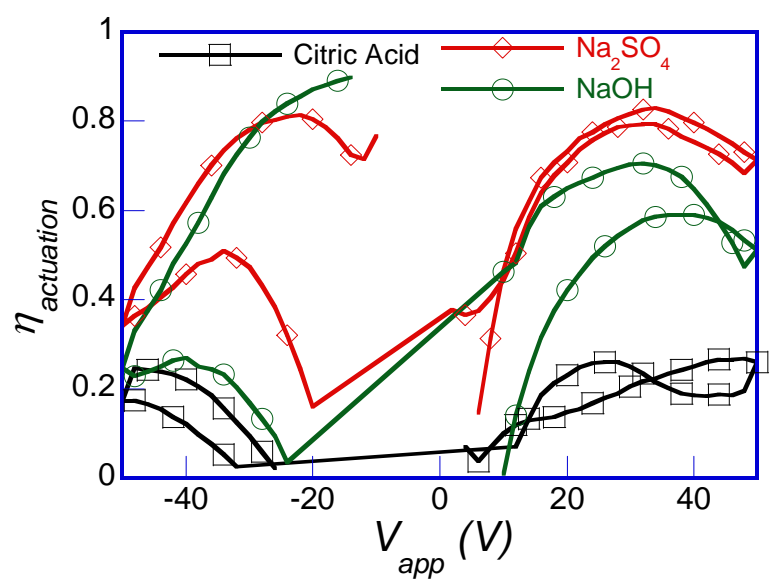

Figure 6. Actuation coefficient versus voltage plot. In this figure, to obtain actuation coefficient values, the data in Figure 5a are used as $V_{\text {left }}$ or $V_{\text {drop }}$.

All fluids show significant deviations from the ideal case (i.e. when $\eta_{\text {actuation }}$ is equal to one at all $\left.V_{a p p}\right)$. Citric acid shows the lowest values, and increasing and decreasing voltages behave very differently. The low $\eta_{\text {actuation }}$ is attributed to a relatively small difference between $V_{\text {left }}$ and $V_{\text {right }}$. With citric acid electrolyte, aluminum oxidation results in a non-porous alumina formation (35), thereby impeding electrolyte diffusion at both cathodic and anodic potential polarities of the electrode. This is seen as a high resistance, which results in the poor behavior observed in electrode pair experiments.

With $\mathrm{Na}_{2} \mathrm{SO}_{4}$ and $\mathrm{NaOH}, \eta_{\text {actuation }}$ increases, yet it is not ideal. There is substantial asymmetry with respect to $V_{a p p}$ polarity and variation with voltage magnitude. This suggests that during initial voltage application (positive) one or both diodes are changed irreversibly. There is also a large hysteresis between increasing and decreasing voltage performance. After electrode pair tests with aluminum $/ \mathrm{Na}_{2} \mathrm{SO}_{4}$ and aluminum $/ \mathrm{NaOH}$ aluminum etching is visible in the test samples. In CEW, this could quickly damage the thin aluminum spots and eliminate the diode behavior. Therefore, these aluminum/electrolyte combinations are inadequate for a reliable CEW device. 
The impact of a preformed alumina layer on the performance was tested by first anodizing an aluminum substrateelectrode at $+50 \mathrm{~V}$ in $0.1 \mathrm{M}$ citric acid bath, which creates an alumina layer approximately $70 \mathrm{~nm}$ thick (36). A $50 \mathrm{~nm}$ Cytop is then applied by spin coating as before. The pair electrode tests were repeated and the results are shown in Figure 7.
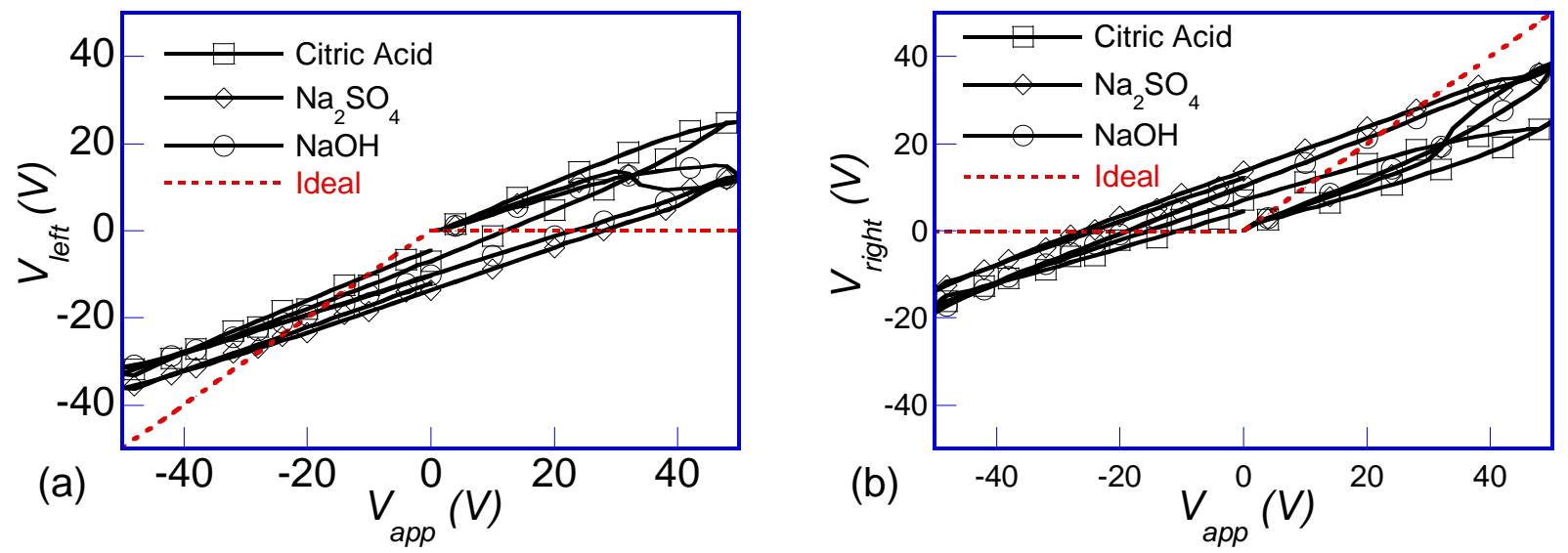

Figure 7. Impact of electrodes pre-anodization on the (a) $V_{\text {left }}$ and (b) $V_{\text {right }}$ behavior in the electrode pair tests. The $V_{\text {left }}$ and $V_{\text {right }}$ are plotted versus the applied voltage. Aluminum is used as electrode with 0.1 $\mathrm{M}$ citric acid, $0.1 \mathrm{M} \mathrm{Na}_{2} \mathrm{SO}_{4}, 0.1 \mathrm{M} \mathrm{NaOH}$ as electrolyte solutions.

Pre-anodized electrodes show poor CEW actuation behavior with nearly linear voltage response. The alumina layer increases the resistance of the forward biased substrateselectrodes at cathodic potentials, so $V_{\text {left }}$ behavior deviates from ideal case as shown in Figure 7a. Therefore, pre-anodization of the metallic spots will not enhance CEW.

\subsubsection{Results with Ti}

The electrode material also influences $\eta_{\text {actuation. }}$ To investigate the effect of the conductive layer material, we repeated the tests using titanium electrodes. The results are shown in Figure 8a and b. Titanium shows much less hysteresis and closer approximations of the ideal diode 
behavior for all electrolytes compared to the aluminum (Figure 6Figure 6). Better clarity is seen in the plot of $\eta_{\text {actuation }}$ versus $V_{a p p}$ as shown in Figure 9 Figure 9.
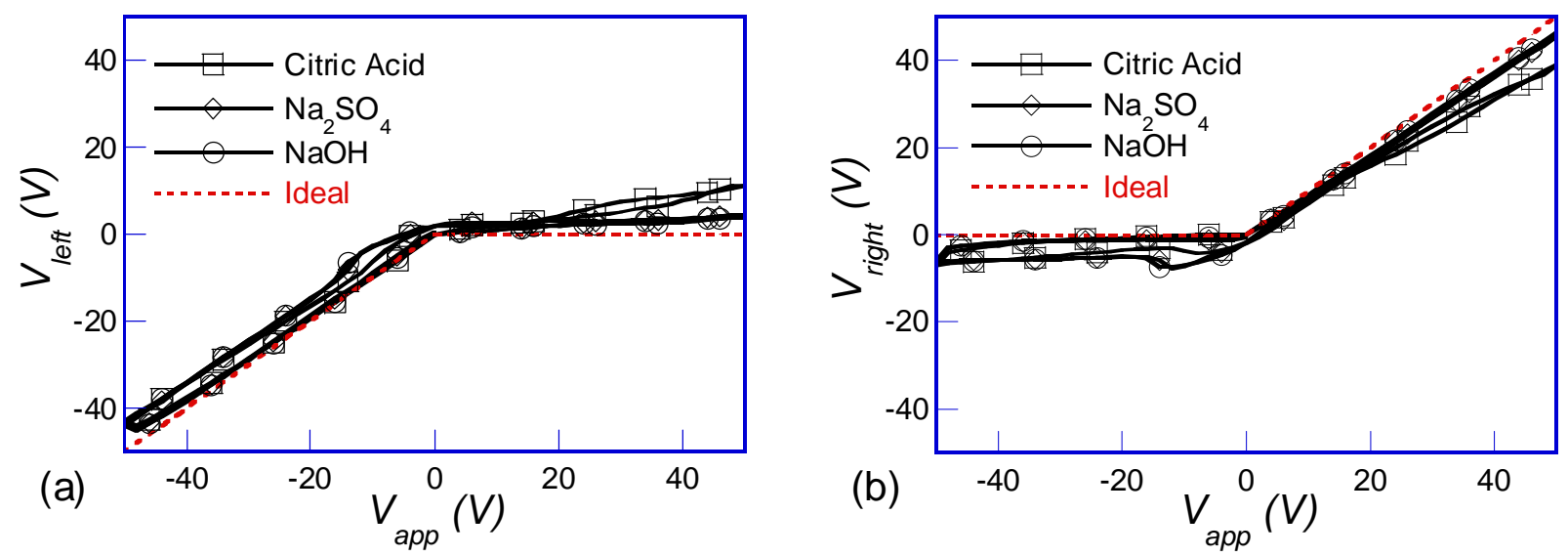

Figure 8. Impact of electrodes material on (a) $V_{\text {left }}$ and (b) $V_{\text {right }}$ in the electrode pair tests. The $V_{\text {left }}$ and $V_{\text {right }}$ are plotted versus the applied voltage. The dashed red lines show the simulated $V_{\text {left }}$ and $V_{\text {right }}$ behavior when the diodes are ideal. $0.1 \mathrm{M}$ citric acid, $0.1 \mathrm{M} \mathrm{Na}_{2} \mathrm{SO}_{4}, 0.1 \mathrm{M} \mathrm{NaOH}$ are used as electrolyte solutions.

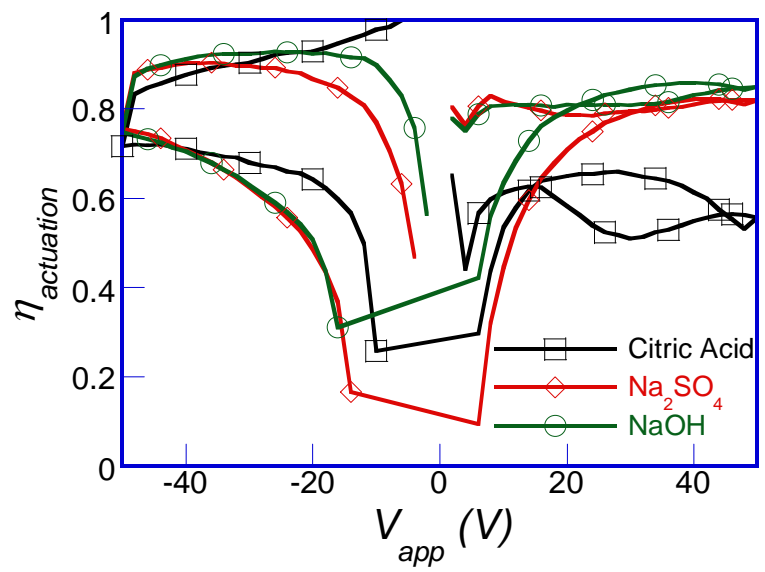

Figure 9. Actuation coefficient versus voltage plot of titanium electrode. In this figure, to obtain actuation coefficient values, the data in Figure 8a are used as $V_{\text {left }}$ or $V_{\text {drop. }}$.

As shown in Figure 9Figure 9, the $\eta_{\text {actuation }}$ values for titanium are substantially higher than for aluminum with titanium $/ \mathrm{Na}_{2} \mathrm{SO}_{4}$ and titanium/ $\mathrm{NaOH}$ combinations significantly higher than 
titanium/citric acid combination. We attribute the high $\eta_{\text {actuation }}$ values with titanium to the stability of titanium oxide during the electrode pair measurement.

It has been argued that titanium oxide formation is accompanied by an inward movement of oxygen from electrolytes solution towards the metal/oxide interface, which makes titanium oxide more protective than the oxides formed upon outward movements of the metal cations (37-38). More recent studies have indicated the concurrent contribution of electrolytes anions and metal cations in the formation of both barrier (protective) and porous oxides, but anion concentrations are lower in barrier oxides than in porous oxides yet the structure of barrier oxides contain at lower anion content than that of porous oxides-(35). In this study, the improved performance of titanium relative to aluminum electrodes may also be related to the differences in their oxidation characteristics.

Typically, in the first trials of the electrode pair experiments, electrode/electrolyte combinations with aluminum result in high values of $\eta_{\text {actuation }}$ (except with aluminum/citric acid), and then over repeated trials there is a significant drop in $\eta_{\text {actuation }}$ values. With titanium, however, $\eta_{\text {actuation }}$ values increase over the initial trials. which-This is attributed to the stabilization of titanium oxide films. Figure 10 shows $V_{\text {left }}$ curves at trials 1,100 , and 500 with aluminum (Figure 10a) and titanium (Figure 10b). At $-50 \mathrm{~V}$, there is clear degradation in aluminum diode behavior with increased cycling, while the diode behavior improves with repeated cycles in the titanium electrodes. Thus, the current/voltage data measured for a first cycle in the titanium electrodes (Figure 4) is not indicative of the service performance under repeated cycling. 

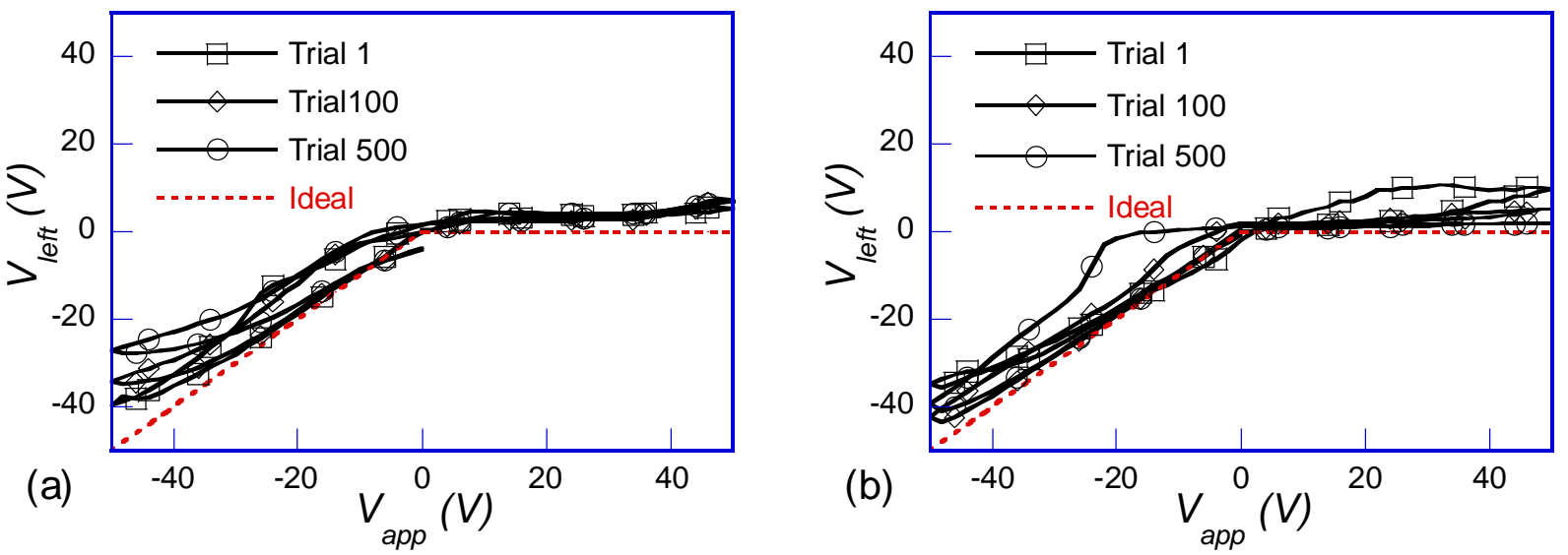

Figure 10. $V_{\text {left }}$ at trials 1, 100, and 500 with (a) aluminum and (b) titanium. The dashed red lines show the simulated $V_{\text {left }}$ and $V_{\text {right }}$ behavior when the diodes are ideal. $0.1 \mathrm{M} \mathrm{Na}_{2} \mathrm{SO}_{4}$ is used as electrolyte solutions.

Electrical current during the electrode pair measurements can also indicate the state of the electrode oxidation. Figure 11 shows the measured current corresponding to Figure 10. The current remains nearly constant with aluminum electrodes (Figure 10a), but with titanium (Figure 10b) the current at the first trial is higher than the 100 and 500 trials. This suggests stabilization of titanium oxide.
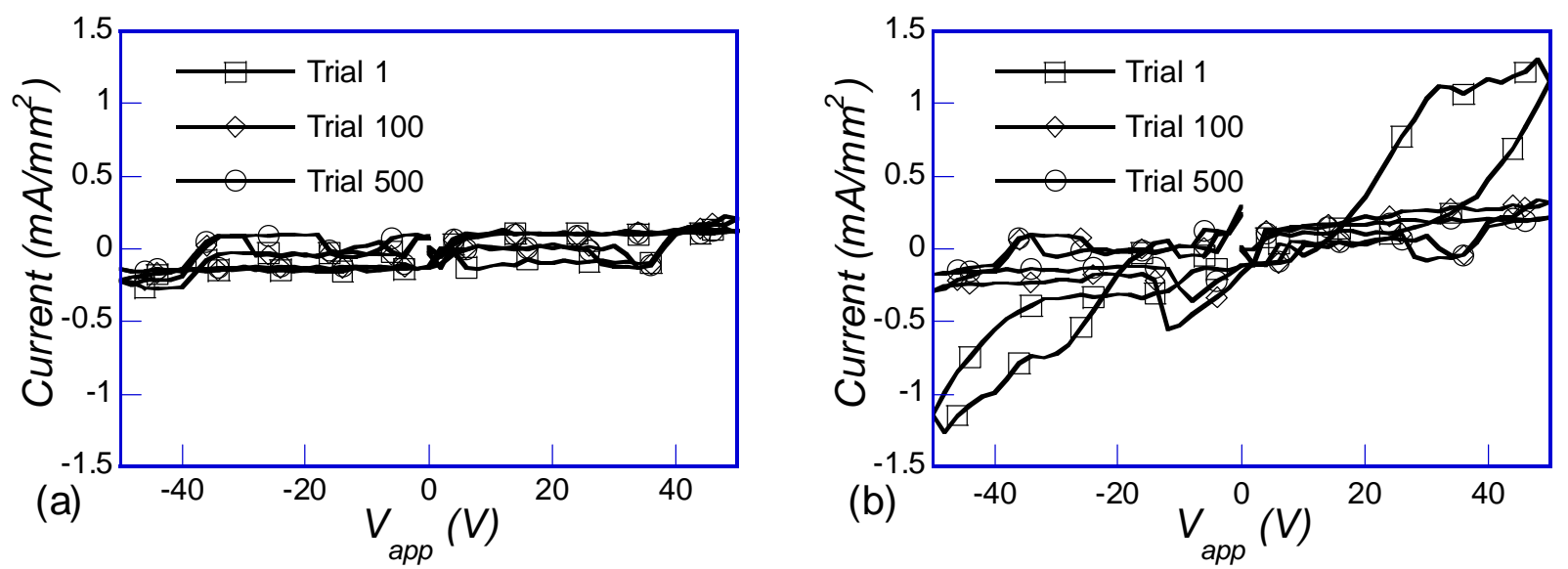

Figure 11. Measured current of the electrode pair measurements with (a) aluminum and (b) titanium electrodes with $0.1 \mathrm{M} \mathrm{N}_{2} \mathrm{SO}_{4}$. 
To evaluate the consistency of the $\eta_{\text {actuation }}$ values over repeated trials, we performed the electrode pair measurements for 500 and 2000 trials respectively with aluminum and titanium electrodes (with the three electrolyte solutions of $0.1 \mathrm{M}$ citric acid, $0.1 \mathrm{M} \mathrm{N}_{2} \mathrm{SO}_{4}, 0.1 \mathrm{M} \mathrm{NaOH}$ ). Figure 12 shows the values of $\eta_{\text {actuation }}$ at the extremes of applied voltage $(+50 \mathrm{~V}$ and $-50 \mathrm{~V})$ for each cycle.
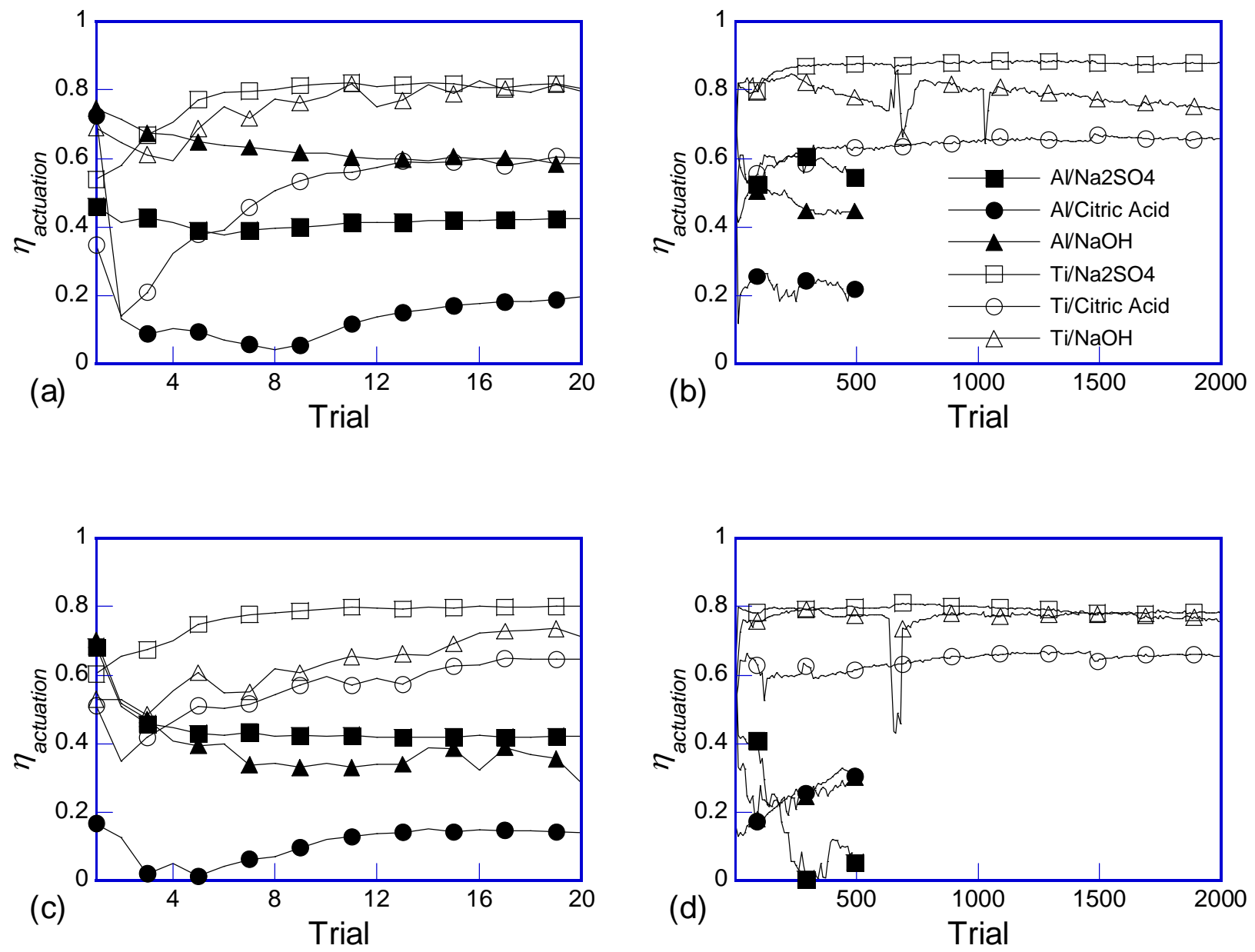

Figure 12. Evaluation of actuation coefficient $\left(\eta_{\text {actuation }}\right)$ reliability in the electrode pair tests for six electrode/electrolyte combinations, namely $\mathrm{Al} / \mathrm{Na}_{2} \mathrm{SO}_{4}, \mathrm{Al} / \mathrm{Citric}$ Acid, $\mathrm{Al} / \mathrm{NaOH}, \mathrm{Ti} / \mathrm{Na}_{2} \mathrm{SO}_{4}, \mathrm{Ti} / \mathrm{Citric}$ Acid, and $\mathrm{Ti} / \mathrm{NaOH}$. Figure 12 shows the $\eta_{\text {actuation }}$ values at the extremes of applied voltage of $+50 \mathrm{~V}(\mathrm{a}, \mathrm{b})$ and $-50 \mathrm{~V}(\mathrm{c}, \mathbf{d})$. Figure $12(\mathrm{a}, \mathrm{c})$ show the $\eta_{\text {actuation }}$ values over the first 20 trials and (b,d) over the full 2000 trials.

In the first trials of the electrode pair measurements with aluminum/ $\mathrm{Na}_{2} \mathrm{SO}_{4}$ and aluminum $/ \mathrm{NaOH}$ combinations, the $\eta_{\text {actuation }}$ values are above 0.6 except for $\mathrm{Al} / \mathrm{Na}_{2} \mathrm{SO}_{4}$ at $-50 \mathrm{~V}$ 
(Figure 12a) and they significantly decrease after repeated trials (Figure 12b). In these measurements, the reduction of the $\eta_{\text {actuation }}$ values is attributed to the instability and etching of aluminum oxide during the electrode pair measurements. This decreases the aluminum electrode resistance when they are reverse biased. The aluminum/citric acid combination is an exception because the $\eta_{\text {actuation }}$ values are low from the very first trials (Figure 12a) and stay low (Figure 12b). which This is attributed to the immediate aluminum passivation. Generally, with aluminum electrodes., the $\eta_{\text {actuation }}$ behavior over repeated trials shows that the CEW devices with aluminum spots would perform appropriately in the beginning, but their performance degrades noticeably after just a few cycles.

However, the reliability of the CEW devices can be improved by the use of titanium spots. As shown in (Figure 12a), with titanium the $\eta_{\text {actuation }}$ values start from around 0.5 but and even increase significantly over the first 10 trials and remain steady (Figure 12b). The improvement of the $\eta_{\text {actuation }}$ values is attributed to a gradual formation of a stable titanium oxide. The titanium electrodes show no visible signs of damage after completing the 2000 voltage cycles. In conclusion, in the beginning, the CEW devices made with aluminum spots might perform as well as those made with titanium spots; however, after repeated use, it is expected that aluminum spot performance would degrade, while titanium spots would keep actuating droplets consistently.

\section{Conclusion}

The voltage across metal/electrolyte contacts are theoretically related to the electrowetting force moving the droplets laterally in CEW. An equation was developed that relates the total force to the droplet radius, applied voltage, and droplet voltage (which is measured experimentally). To evaluate the performance of different electrode/electrolyte combinations, an electrode pair experiment was designed, and then the experimental results were related to the 
metallic spot performance in real CEW devices via a coefficient which here is-referred to as the actuation coefficient $\left(\eta_{\text {actuation }}\right)$. The $\eta_{\text {actuation }}$ values in six electrode/electrolyte combinations, namely $\mathrm{Al} / \mathrm{Na}_{2} \mathrm{SO}_{4}, \mathrm{Al} /$ citric acid, $\mathrm{Al} / \mathrm{NaOH}, \mathrm{Ti} / \mathrm{Na}_{2} \mathrm{SO}_{4}, \mathrm{Ti} /$ citric acid, and $\mathrm{Ti} / \mathrm{NaOH}$ were evaluated.

The $\eta_{\text {actuation }}$ magnitudes in $\mathrm{Al} / \mathrm{NaOH}$ and $\mathrm{Al} / \mathrm{Na}_{2} \mathrm{SO}_{4}$ combinations are higher than those in Al/citric acid, but they vary with voltage. In the repeated electrode pair measurements with aluminum, in the first trials, the $\eta_{\text {actuation }}$ values are high for all but the $\mathrm{Al} /$ citric acid combination. In this combination, the $\eta_{\text {actuation }}$ values are low from the very first trial, which is attributed to the immediate passivation of aluminum. Once passivated, the electrodes impede the ions diffusion at both anodic and cathodic polarities of the electrode, reducing $\eta_{\text {actuation }}$ in $\mathrm{Al} /$ citric acid combinations. Aluminum electrode performance declined significantly with repeated cycling, which is possibly due to the alumina etching. In general, aluminum spots might perform well in the first trials, but a significant degradation in their performance is expected.

With titanium electrodes, all of the $\eta_{\text {actuation }}$ values approached 0.8 after the initial 10 cycles, significantly higher than those obtained with the aluminum electrode. However, with citric acid the $\eta_{\text {actuation }}$ values were slightly lower than for the other electrolytes at positive applied voltages. This could be attributed to a high resistance of the titanium oxide (formed with citric acid) against electrolyte diffusion. With the titanium electrode, another aspect of the $\eta_{\text {actuation }}$ curves is that they do not decrease around the extreme voltages (unlike the $\eta_{\text {actuation }}$ curves with the aluminum electrodes). The consistency of the $\eta_{\text {actuation }}$ curves can be related to the stability of titanium oxide over the electrode pair measurements. In the repeated electrode pair measurements, titanium electrodes showed consistent performance over 2000 cycles, which is 
also attributed to the titanium oxide stability. With the use of titanium spots, we can fabricate reliable devices that utilize electrochemical diodes such as the CEW devices discussed here.

\section{Acknowledgements}

This work was supported in part by the National Science Foundation through grant CMMI1130755.

\section{References}

1. K. G. Hans-Jürgen Butt, M. Kappl, Physics and Chemistry of Interfaces, John Wiley \& Sons (2003).

2. J. B. Hudson, Surface Science: An Introduction, John Wiley \& Sons (1998).

3. S. W. Rienstra, J Eng Math, 24, 193 (1990).

4. C. Cooney, C.-Y. Chen, M. Emerling, A. Nadim and J. Sterling, Microfluid. Nanofluid., 2, 435 (2006).

5. $\quad$ V. A. Lubarda and K. A. Talke, Langmuir, 27, 10705 (2011).

6. T. B. Jones, J. Micromech. Microeng. (2005).

7. K. H. Kang, Langmuir, 18, 10318 (2002).

8. F. Mugele and J. C. Baret, J. Phys.: Condens. Matter, 17, 705 (2005).

9. C. E. Rosenkilde, Proc. R. Soc. London, Ser. A, 312, 473 (1969).

10. T. B. Jones, J. D. Fowler, Y. S. Chang and C.-J. Kim, Langmuir, 19, 7646 (2003).

11. J. Buehrle, S. Herminghaus and F. Mugele, Phys. Rev. Lett., 91, 86101 (2003).

12. F. Mugele and J. Buehrle, J. Phys.: Condens. Matter, 19, 375112 (2007).

13. J. T. Cheng and C. L. Chen, Nanoscale Microscale Thermophys. Eng., 14, $63(2010)$.

14. C. Knospe and S. Nezamoddini, J. Micro-Nano Mech., 5, 57 (2009).

15. B. Berge and J. Peseux, Eur. Phys. J. E, 3, 159 (2000).

16. F. Mugele and J.-C. Baret, J. Phys.: Condens. Matter, 17, R705 (2005).

17. B. H. W. Hendriks, S. Kuiper, M. A. J. As, C. A. Renders and T. W. Tukker, Opt. Rev., 12, 255 (2005).

18. R. A. Hayes and B. J. Feenstra, Nature, 425, 383 (2003).

19. R. Shamai, D. Andelman, B. Berge and R. Hayes, Soft Matter, 4, 38 (2008).

20. T. Krupenkin and J. A. Taylor, Nat. Commun., 2, 448 (2011). 
21. M. Abdelgawad and A. R. Wheeler, Adv. Mater., 19, 133 (2007).

22. C. Cooney, C.-Y. Chen, M. Emerling, A. Nadim and J. Sterling, Microfluid. Nanofluid., 2, 435 (2006).

23. M. W. L. Watson, M. Abdelgawad, G. Ye, N. Yonson, J. Trottier and A. R. Wheeler, Anal. Chem., 78, 7877 (2006).

24. K. P. Nichols and J. G. E. Gardeniers, Anal. Chem., 79, 8699 (2007).

25. E. M. Miller and A. R. Wheeler, Anal. Chem., 80, 1614 (2008).

26. P. Paik, V. K. Pamula and R. B. Fair, Lab Chip, 3, 253 (2003).

27. E. Miller and A. Wheeler, Anal Bioanal Chem, 393, 419 (2009).

28. C. Sung Kwon, M. Hyejin and K. Chang-Jin, J. Microelectromech. Syst., 12, 70 (2003).

29. M. Khodayari, N. B. Crane and A. A. Volinsky, Thin Solid Films, 548, 632 (2013).

30. N. B. Crane, A. a. Volinsky, P. Mishra, A. Rajgadkar and M. Khodayari, Appl. Phys. Lett., 96, 104103 (2010).

31. C. W. Nelson, C. M. Lynch and N. B. Crane, Lab Chip, 11, 2149 (2011).

32. S. Chevalliot, S. Kuiper and J. Heikenfeld, J. Adhes. Sci. Technol., 26, 1909 (2012).

33. M. Dhindsa, J. Heikenfeld, W. Weekamp and S. Kuiper, Langmuir, 27, 5665 (2011).

34. M. Khodayari, B. Hahne, N. B. Crane and A. A. Volinsky, Appl. Phys. Lett., $102(2013)$.

35. G. D. Sulka and A. Eftekhari, Nanostruct. Mater. Electrochem. (2008).

36. S. Wernick, R. Pinner and P. G. Sheasby, The surface treatment and finishing of aluminum and its alloys, in, ASM International, Finishing Publication Ltd. (1987).

37. U. R. Evans, The Corrosion and Oxidation of Metals, Edward Arnold, London (1960).

38. L. L. Shreir, Corrosion. Vol. 1. Metal/Environment Reactions, Butterworth \& Co. (Publishers) Ltd. (1976). 